\title{
Geometrically frustrated Ising-Heisenberg spin model on expanded Kagomé lattice
}

\author{
Onofre Rojas \\ Departamento de Física, Universidade Federal de Lavras, CP 3037, 37200-000, MG, Brazil
}

\begin{abstract}
Here we consider the Ising-Heisenberg model in the expanded Kagomé lattice, also known as triangle-dodecagon (3-12) or star lattice. This model can still be understood as a decorated honeycomb lattice. Assuming that the Heisenberg spins are at the vertices of the triangle while other spins are of the Ising type. Thus, this model is equivalent to an effective Kagomé Ising lattice, through the decoration transformation technique. Thus this means that the model is exactly solvable so we can study the most relevant properties of this model. Like the phase diagram at zero temperature, exhibiting a frustrated phase, a ferromagnetic phase, a classical ferrimagnetic phase and a quantum ferrimagnetic phase. We observed that Heisenberg spin exchange interaction influences the frustrated phase, but we rigorously verify that the magnitude and origin of the frustration emerge in a similar way to antiferromagnetic Ising Kagomé lattice. Likewise, the thermodynamic properties of the model can also be obtained, such as the critical temperature as a dependence of the Hamiltonian parameters and the spontaneous magnetization of the model. Besides, we investigated the entropy of the model, identifying its residual entropy in the frustrated region. Even we analyze the specific heat behavior as a temperature dependence, to deal with the phase transition.
\end{abstract}

Keywords: Exactly solvable modes, Decoration transformation, Kagomé lattice, 2D Ising model, IsingHeisenberg model.

\section{INTRODUCTION}

One of the most relevant topics in statistical physics is the search for exactly solvable models. In general, spin models in statistical physics can not be solved exactly, so most of them can only be studied numerically. Thus, the exact solutions were obtained only for limited cases. After the solution found by Onsager for two-dimensional Ising model[1], it inspired several attempts to solve other similar models. Such as the honeycomb lattice [2, 3], whose exact solution of a honeycomb lattice with an external magnetic field was provided by $\mathrm{Wu}[4]$. Besides, Kagomé lattice was also widely discussed in the literature [5, 6] and reference there in.

Typically geometric frustration arises in spin triangular structures. When the competing antiferromagnetic interactions cannot be satisfied simultaneously, leading to a considerable degeneracy of ground states. In such a way, frustrated magnets have been attracted great scientific interest because of quantum spin liquid in twodimensional systems, which has been proposed to play a striking role in high-temperature superconductors. Furthermore, theoretical investigations have confirmed that spin-1/2 Kagomé compounds are one of the natural candidates for obtaining a quantum spin liquid ground state. This means, due to the strong quantum fluctuation and geometric frustration would be responsible for extinguishing the classical long-range magnetism at low temperature.

The importance of investigations for geometrically frustrated kagomé lattice compound is a great challenge. But there are few materials with spin- $1 / 2$ kagomé structure that exhibit the quantum spin liquid state in zero temperature. One of the typical illustrative examples could be $\mathrm{ZnCu}_{3}(\mathrm{OH})_{6} \mathrm{Cl}_{2}[7,8]$, which shows a regular kagomé lattice. Using the single crystal sample of $\mathrm{ZnCu}_{3}(\mathrm{OH})_{6} \mathrm{Cl}_{2}$ were observed several relevant characteristics of the quantum spin liquid phase. Besides, investigations of spin- $1 / 2$ systems are quite relevant in another context. Such discussed the magnetic properties through canonical ensemble thermodynamic potentials of magnetic systems [9]. And the study of spin- $1 / 2$ bilayer system with the Glauber-type stochastic dynamic behavior using the effective-field theory approach $[10]$.

Several decorated spin models can be transformed by applying the well-known decoration transformation established in the 1950's by M. E. Fisher[11] and Syozi [12]. Later generalized in reference [13-15], for arbitrary spins, such as the classical or quantum spin models. This transformation is essential because we can map cumbersome models into a simple or exactly solvable models. Below we mention few typical examples where this approach was successfully applied. Geometrical frustrated Cairo pentagonal lattice Ising model[16]. The Blume-Emery-Griffiths (BEG) [17] model on the honeycomb lattice, further investigated by Horiguchi[2], Wu [18], Tucker[19] and Urumov 20], applying the standard decoration transformation[11, 12] and satisfying the Horiguchi's condition[2]. As well as XXZ-Ising model on the triangular Kagomé lattice with spin- $1 / 2$, was studied using analytical 21, 22] and Monte Carlo simulations [21].

On the other hand low-dimensional square-hexagon (denoted for simplicity by 4-6) Ising with spin- $1 / 2$ model was discussed by Lin and Chan 23] using the eight-vertex models mapping, later generalized as XXZ-Ising model on a square-hexagon (4-6) lattice with spin-1/2, which was investigated using the same approach $26 \mid$. Similarly, the 3-12 lattice also known in the literature [24, 25], as the star lattice, Fisher lattice, expanded Kagomé lattice, or even triangular honeycomb lattice. Motivated the study of this kind of model due to its closely relation with geometrically frustrated magnetic material polymers [27].

The present work is organized as follows. In Sec. 2, we 
present the expanded Kagomé Ising-Heisenberg model. In Sec. 3, we discuss the phase transition at zero temperature. While in Sec. 4, we give the mapping of expanded Kagomé Ising-Heisenberg model into the Kagomé Ising model. Furthermore, in Sec. 5, we discuss the thermodynamics of the models, such as critical temperature, spontaneous magnetization entropy, and specific heat. Finally, in sec. 6 we offer our conclusions.

\section{EXPANDED KAGOMÉ LATTICE HAMILTONIAN}

The Ising-Heisenberg expanded Kagomé lattice is built up by triangles and dodecagons (3-12). Where thick solid line in triangles structure between small circles represents the Heisenberg spin exchange bonds (see Fig. 1). Likewise, the remaining couplings (thin solid line) connecting small and large circles correspond to Ising type exchange interactions as illustrated in Fig 1. This lattice can even be identified as a decorated star lattice[27, 28], which synthesizes a chemical compound. Besides, Fig:1 illustrates the unit cell of expanded Kagomé lattice by a parallelogram. Thus, each unit cell is composed of 3 Heisenberg spins and 6 Ising spins.

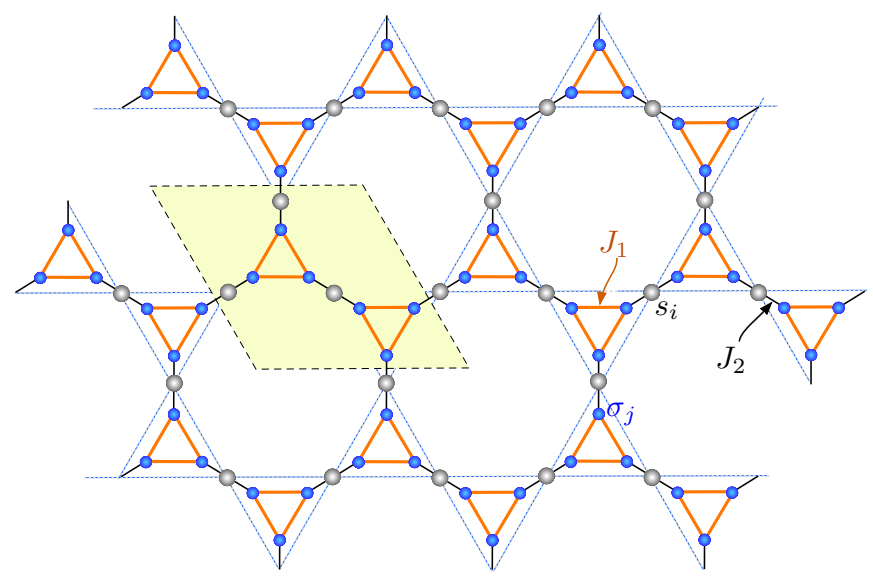

Figure 1: Schematic lattice representation of expanded Kagomé Ising-Heisenberg model. Small circles represent the Heisenberg spins, while large circles denote the Ising spins, thick lines represent the Heisenberg coupling while thin lines correspond to the Ising coupling. The parallelogram is symbolizing the expanded Kagomé lattice unit cell.

The Hamiltonian that describes the expanded Kagomé lattice can be expressed by

$$
H=-J_{1} \sum_{<i, j>}\left(\boldsymbol{\sigma}_{i}, \boldsymbol{\sigma}_{j}\right)_{\Delta}-J_{2} \sum_{<k, l>} \sigma_{k}^{z} s_{l},
$$

here by $\sigma^{\alpha}$ we denote the Heisenberg spins operator with $\alpha=\{x, y, z\}$, whereas $s_{i}$ denotes the Ising spin $s_{i}= \pm 1 / 2$. The first summation corresponds to the anisotropic Heisenberg coupling between the nearest

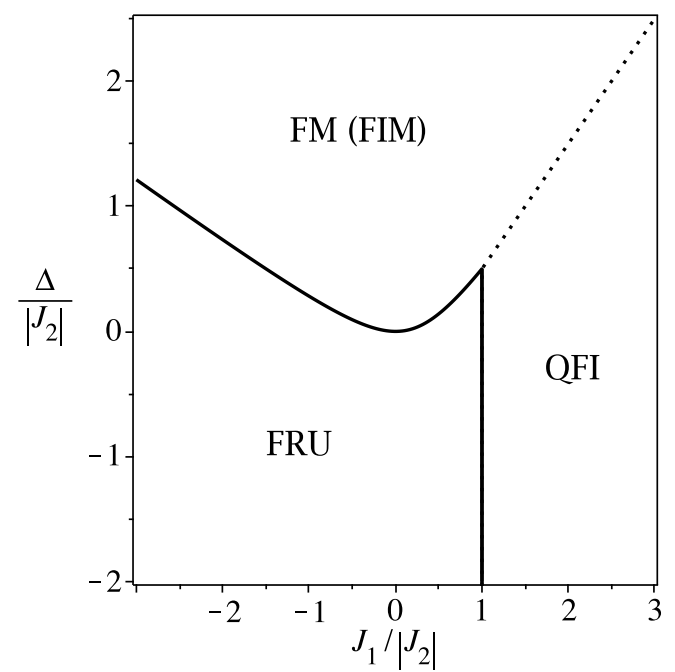

Figure 2: Zero temperature phase diagram in the plane $J_{1} /\left|J_{2}\right|-\Delta /\left|J_{2}\right|$.

neighbor, which is explicitly given by

$$
J_{1}\left(\boldsymbol{\sigma}_{i}, \boldsymbol{\sigma}_{j}\right)_{\Delta}=J_{1}\left(\sigma_{i}^{x} \sigma_{j}^{x}+\sigma_{i}^{y} \sigma_{j}^{y}\right)+\Delta \sigma_{i}^{z} \sigma_{j}^{z},
$$

where $J_{1}$ is the Heisenberg exchange interaction in $x y$ component and $\Delta$ correspond to Heisenberg exchange interaction in $z$ component.

While the second summation represents the sum of the nearest Ising and Heisenberg spins coupling and $J_{2}$ denotes the Ising spin exchange interaction parameter.

\section{PHASE DIAGRAM}

The phase diagram at zero temperature is illustrated in units of $\left|J_{2}\right|$ as shown in Fig 2. Thus the diagram exhibits a ferromagnetic $(\mathrm{FM})$ phase for $J_{2}>0$ and a ferrimagnetic (FIM) phase for $J_{2}<0$, with the corresponding ground state energy per unit cell given by

$$
E=-\frac{3\left|J_{2}\right|}{2}-\frac{3 \Delta}{2}, \text { with } \begin{cases}F M ; & \text { for } J_{2}>0 \\ F I M ; & \text { for } J_{2}<0\end{cases}
$$

This ground state configuration per unit cell is schematically depicted in Fig $[3$, where the corresponding eigenstates can also be expressed as follows

$$
\begin{aligned}
|F M\rangle & \left.\left.=\left.\right|_{+} ^{+}\right\rangle\left.\otimes\right|_{+} ^{+} \triangleright+\right\rangle \otimes|+\rangle \otimes\left|+\triangleleft_{+}^{+}\right\rangle, \\
|F I M\rangle & \left.\left.=\left.\right|_{+} ^{+}\right\rangle\left.\otimes\right|_{-} ^{-} \triangleright-\right\rangle \otimes|+\rangle \otimes\left|-\triangleleft_{-}^{-}\right\rangle .
\end{aligned}
$$

Clearly, (44) and (5) are invariant under total spin inversion.

The total magnetization per unit cell of expanded Kagomé lattice is given by

$$
m_{t}=\frac{m_{I}+2 m_{H}}{3},
$$


(a)
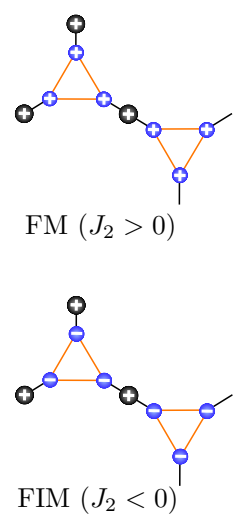

(b)
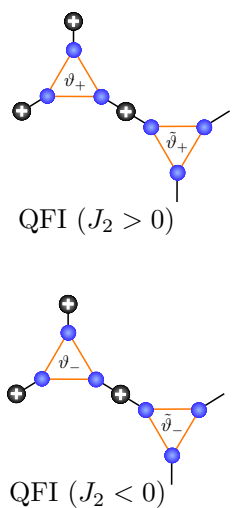

(c)
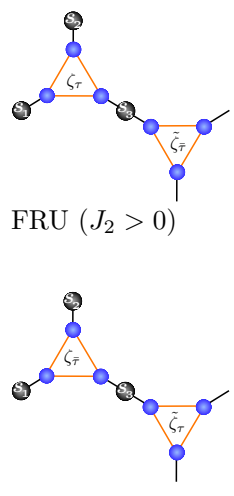

$\operatorname{FRU}\left(J_{2}<0\right)$
Figure 3: Ground state configurations per unit cell. (a) Classical Ferromagnetic (Ferrimagnetic), (b) quantum ferrimagnetic (QFI) phase and in (c) frustrated (FRU) phase.

with $m_{I}$ being the Ising spins magnetization per Ising spin sites, and $m_{H}$ denotes the Heisenberg spins magnetization per Heisenberg spin sites.

Using this setting, the Ising spin magnetization is given by $m_{I}=1 / 2$ and the Heisenberg spin magnetization is $m_{H}=1 / 2$. Therefore, for $J_{2}>0$ arises the FM phase (here we denote $s=\sigma=+$ ) with a total magnetization per unit cell is $m_{t}=1 / 2$.

Whereas for $J_{2}<0$ the system is in FIM phase $(s=-\sigma=+$, by + we mean $+1 / 2)$ with Ising spin magnetization $m_{I}=1 / 2$, while Heisenberg spin magnetization is $m_{H}=-1 / 2$ and the total magnetization per unit cell becomes $m_{t}=-1 / 6$.

The other state illustrated in Fig $[3$ is the quantum ferrimagnetic (QFI) phase, which is represented schematically in Fig 3 b. This state can be written explicitly as follows,

$$
|Q F I\rangle=\left|{ }_{s}^{s}\right\rangle \otimes\left|\vartheta_{\sigma}\right\rangle \otimes|s\rangle \otimes\left|\tilde{\vartheta}_{\sigma}\right\rangle,
$$

with

$$
\begin{aligned}
& \left|\vartheta_{\sigma}\right\rangle=\frac{1}{\sqrt{3}}\left(\left|{ }_{\sigma}^{\sigma} \triangleright \bar{\sigma}\right\rangle+\left|{ }_{\sigma}^{\bar{\sigma}} \triangleright \sigma\right\rangle+\left|{ }_{\bar{\sigma}}^{\sigma} \triangleright \sigma\right\rangle\right), \\
& \left|\tilde{\vartheta}_{\sigma}\right\rangle=\frac{1}{\sqrt{3}}\left(\left|\bar{\sigma} \triangleleft_{\sigma}^{\sigma}\right\rangle+\left|\sigma \triangleleft_{\bar{\sigma}}^{\sigma}\right\rangle+\left|\sigma \triangleleft_{\sigma}^{\bar{\sigma}}\right\rangle\right),
\end{aligned}
$$

here, by $\bar{\sigma}$ we mean $\bar{\sigma}=-\sigma$. A particular case of QFI state becomes

$$
|Q F I\rangle=\left\{\begin{array}{ll}
\left.\left.\right|_{+} ^{+}\right\rangle \otimes\left|\vartheta_{+}\right\rangle \otimes|+\rangle \otimes\left|\tilde{\vartheta}_{+}\right\rangle, & J_{2}>0 \\
\left.\left.\right|_{+} ^{+}\right\rangle \otimes\left|\vartheta_{-}\right\rangle \otimes|+\rangle \otimes\left|\tilde{\vartheta}_{-}\right\rangle, & J_{2}<0
\end{array} .\right.
$$

Since the corresponding ground state energy is given by

$$
E_{Q F I}=-\frac{\left|J_{2}\right|}{2}+\frac{\Delta}{2}-2 J_{1} .
$$

In QFI phase, there are also two possible total magnetizations: (i) When $J_{2}>0$ the spin configuration satisfy $s=\sigma=+$, whose Ising spin magnetization $m_{I}=1 / 2$ and Heisenberg spin magnetization $m_{H}=1 / 6$ and its corresponding total magnetization is $m_{t}=5 / 18$. (ii) When $J_{2}<0$ the spin configuration satisfy $s=-\sigma$, thus the Ising spin magnetization $m_{I}=1 / 2$, Heisenberg spin magnetization $m_{H}=-1 / 6$ and the total magnetization for this configuration is given by $m_{t}=1 / 18$.

In Fig 2 is also reported a frustrated (FRU) phase, at zero temperature. A schematic spin configuration displayed in Fig. 3r, whose state is expressed below

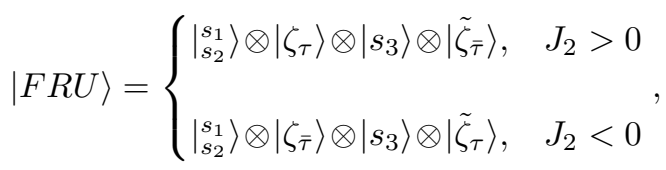

and denoting the Heisenberg spins states by

$$
\begin{aligned}
& \left.\left.\left.\left|\zeta_{\tau}\right\rangle=\frac{1}{\sqrt{2+a^{2}}}\left(\left.a\right|_{\sigma_{2}} ^{\sigma_{1} \triangleright \sigma_{3}}\right\rangle+\left.\right|_{\sigma_{1}} ^{\sigma_{3} \triangleright \sigma_{2}}\right\rangle+\left.\right|_{\sigma_{3}} ^{\sigma_{2} \triangleright \sigma_{1}}\right\rangle\right), \\
& \left|\tilde{\zeta}_{\tau}\right\rangle=\frac{1}{\sqrt{2+a^{2}}}\left(a\left|\sigma_{1} \triangleleft_{\sigma_{2}}^{\sigma_{3}}\right\rangle+\left|\sigma_{3} \triangleleft_{\sigma_{1}}^{\sigma_{2}}\right\rangle+\left|\sigma_{2} \triangleleft_{\sigma_{3}}^{\sigma_{1}}\right\rangle\right) .
\end{aligned}
$$

Where Ising spins are restricted to $s_{1}+s_{2}+s_{3}=\tau$, analogously we have $\sigma_{1}+\sigma_{2}+\sigma_{3}=\tau$, with $\tau$ restricted to $\tau= \pm$ (here $\pm 1 / 2$ ), and defining for convenience $\bar{\tau}=$ $-\tau$. The frustrated state (12) illustrated in Fig. 3ro are equivalent through Heisenberg spin inversion.

Whereas the coefficients is given by

$$
a=\left\{\begin{array}{ll}
u_{1,+}=-\frac{1}{2}+\frac{J_{2}}{J_{1}}+\frac{J_{-}}{2 J_{1}}, & J_{2}>0 \\
u_{2,+}=-\frac{1}{2}-\frac{J_{2}}{J_{1}}+\frac{J_{+}}{2 J_{1}}, & J_{2}<0
\end{array} .\right.
$$

with $J_{ \pm}=\sqrt{4 J_{2}^{2}+9 J_{1}^{2} \pm 4 J_{1} J_{2}}$ (denoted just for convenience).

The corresponding ground state energy of a frustrated state is given by

$$
E_{F R U}=\left\{\begin{array}{ll}
-\frac{J_{2}}{2}+\frac{\Delta-J_{1}-J_{-}}{2}, & J_{2}>0 \\
\frac{J_{2}}{2}+\frac{\Delta-J_{1}-J_{+}}{2}, & J_{2}<0 .
\end{array} .\right.
$$

It is worth to mention that the magnetizations of Ising spins and Heisenberg spins in the frustrated region are null. So the only one responsible for generating frustration is Ising spins, as discussed in Ref. [29].

A straight line $\Delta /\left|J_{2}\right|=-\frac{1}{2}+J_{1} /\left|J_{2}\right|$ gives the boundary between FM(FIM) phase and QFI phase. It deserves to remark that the interface between (FIM or FM) and QFI only occur at zero temperature. While the boundary between FM(FIM) phase and FRU phase is described by the curve $\frac{\Delta}{\left|J_{2}\right|}=-\frac{1}{2}+\frac{J_{1}+J_{-}}{4\left|J_{2}\right|}$, and this phase transition persist at finite temperature which will be discussed later. Afterward, the phase transition between QFI and FRU is plainly given by a $J_{1} /\left|J_{2}\right|=1$, this interface also remains at finite temperature. 


\section{EXPANDED KAGOMÉ LATTICE ON ISING-HEISENBERG MODEL}

Now let us consider a three-leg hybrid-star system as schematically depicted in Fig 4 (left), where the three Heisenberg spins localized at the vertex of a triangle corresponding to the system decoration. In which the internal bond $J_{1}$ is of the Heisenberg-type interaction since the outer legs are of Ising-type interaction $J_{2}$ as shown in Fig. 4(left side). This system can be mapped in a triangle with the Ising spin coupling $K$ through a direct decoration transformation approach 15 ] or using the standard decoration transformation [11 14], which is shown in Fig. 4(right).
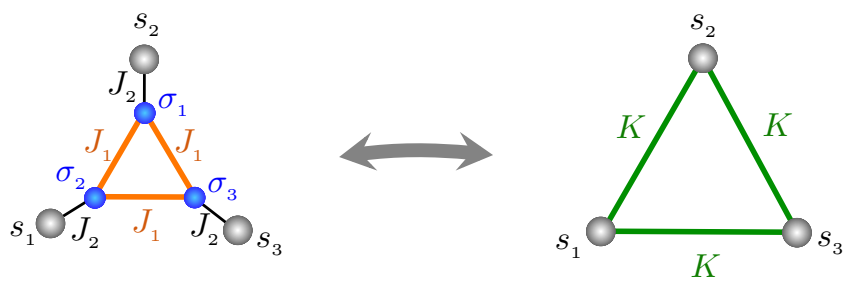

Figure 4: Decoration transformation of 3-leg star hybrid-spin and triangle Ising spin.

Let us define the following operator

$$
\mathbf{V}(\{\boldsymbol{\sigma}, s\})=\exp \left\{\beta \sum_{i=1}^{3}\left[J_{1}\left(\boldsymbol{\sigma}_{i}, \boldsymbol{\sigma}_{i+1}\right)_{\Delta}+J_{2} \sigma_{i}^{z} s_{i}\right]\right\} .
$$

Thus the Boltzmann factors of decorated hybrid-spin model become

$$
w(\{s\})=\operatorname{tr}_{\{\sigma\}}(\mathbf{V}(\{\boldsymbol{\sigma}, s\})),
$$

the index of spin $s_{l}$ in Hamiltonian (1), was re-indexed by $s_{i}$ (same index of $\sigma_{i}$ ) just for simplicity imposing no restriction.

The inner triangle system (decorated) is expressed as Heisenberg coupling, and it provides two configurations for Ising spins (legs), these correspond to the following configurations $\{\uparrow \uparrow \uparrow\}$ and $\{\uparrow \uparrow \downarrow\}$. So defining $\varsigma=s_{1}+$ $s_{2}+s_{3}$, the pair configurations become $\varsigma=3 / 2$ and $1 / 2$. Therefore, we get the following Boltzmann factors defined by $w(1 / 2)=w_{1}$ and $w(3 / 2)=w_{3}$, and then we find that

$$
\begin{aligned}
& w_{1}=\left(z^{3}+z^{-1} x^{-2}\right)\left(y+y^{-1}\right)+x z^{-1}\left(y^{-1} v_{+}+y v_{-}\right), \\
& w_{3}=z^{3}\left(y^{3}+y^{-3}\right)+z^{-1}\left(x^{4}+2 x^{-2}\right)\left(y+y^{-1}\right)
\end{aligned}
$$

Here we have introduced the following notations $x=$ $\mathrm{e}^{\frac{\beta J_{1}}{4}}, y=\mathrm{e}^{\frac{\beta J_{2}}{4}}, z=\mathrm{e}^{\frac{\beta \Delta}{4}}$ and $v_{ \pm}=2 \cosh \left(\frac{\beta J_{ \pm}}{4}\right)$, just as a shorthand way of writing the Boltzmann factors.

Now let us assume that the Hamiltonian of effective Kagomé lattice Ising model can be expressed as follows

$$
\mathcal{H}_{e f f}=-K_{0}-K \sum_{<i, j>} s_{i} s_{j}
$$

where $K_{0}$ is "constant" energy and $K$ is effective coupling parameter of the Kagomé lattice Ising model, while the summation runs over nearest neighbor interactions.

After carrying out the direct decoration transformation [15], the effective Kagomé lattice Ising model also has only the same couple of configurations $\{\uparrow \uparrow \uparrow\}$ and $\{\uparrow \uparrow \downarrow\}$, which correspond to $\varsigma=3 / 2$ and $\varsigma=1 / 2$, respectively. Therefore, both models must be equivalent. That means $\tilde{w}(\varsigma)=w(\varsigma)$, we have two algebraic equations with two unknown parameters $K_{0}$ and $K$, thus we are able to solve the algebraic system equations,

$$
\begin{gathered}
\mathrm{e}^{\beta K_{0}} \exp (-\beta K / 4)=w_{1}=\tilde{w}_{1}, \\
\mathrm{e}^{\beta K_{0}} \exp (3 \beta K / 4)=w_{3}=\tilde{w}_{3} .
\end{gathered}
$$

Thereafter, the unknown parameters in the effective Kagomé Ising model could be expressed in terms of all arbitrary parameters of the expanded Kagomé IsingHeisenberg spin model [see Eq. (1)],

$$
\begin{aligned}
K & =\frac{1}{\beta} \ln \left(\frac{w_{3}}{w_{1}}\right), \\
K_{0} & =\frac{1}{4 \beta} \ln \left(w_{1}^{3} w_{3}\right),
\end{aligned}
$$

where $w_{1}$ and $w_{3}$ are given by (19) and (20) respectively.

\section{THERMODYNAMICS}

Now we are going to study the thermodynamics of the present model, so we need to get the free energy per unit cell of the model that can be written as

$$
f_{E K}=-2 K_{0}+f_{K}
$$

With $f_{K}$ being the effective Kagomé lattice Ising model free energy $12,29,30$ and $K_{0}$ corresponds to the effective "constant" energy of the effective Kagomé lattice. The factor 2 in $K_{0}$ comes from unit cell, note that we have two decorated systems per each unit cell.

The free energy of effective Kagomé lattice per unit cell [12, 29, 30] can be expressed using a single integral 31], as follows

$$
f_{K}=-\frac{T}{4 \pi} \int_{0}^{2 \pi} \ln [A(\phi)+\sqrt{Q(\phi)}] \mathrm{d} \phi,
$$

where $A(\phi)$ and $Q(\phi)$ are defined by

$$
\begin{aligned}
& A(\phi)=\frac{1}{2} r^{4}+9 r^{2}+12 r+\frac{21}{2}-2(r-1)\left(r^{2}-1\right) \cos (\phi), \\
& Q(\phi)=A(\phi)^{2}-8(r-1)^{2}\left(r^{2}-1\right)^{2}[1+\cos (\phi)],
\end{aligned}
$$

with $r=\frac{w_{3}}{w_{1}}$. Thus, the free energy per unit cell of expanded Kagomé lattice becomes

$$
f_{E K}=-2 T \ln \left(w_{1}\right)-\frac{T}{4 \pi} \int_{0}^{2 \pi} \ln [A(\phi)+\sqrt{Q(\phi)}] \mathrm{d} \phi .
$$


Before continuing studying the thermodynamics properties, we need to remark three interesting points.

First concerning to a residual entropy in FRU region, occurs when $T \rightarrow 0$ and $w_{3}<w_{1}$, this implies that $r=$ $\frac{w_{3}}{w_{1}} \rightarrow 0$, then the elements of integral reduce to

$$
\begin{aligned}
& A(\phi)=2 \cosh (\phi)+\frac{21}{2}, \\
& Q(\phi)=\left[2 \cosh (\phi)+\frac{21}{2}\right]^{2}+8[1+\cos (\phi)],
\end{aligned}
$$

both functions are independent of $T$ and $r$. Therefore, the free energy (28) can be solved numerically for (29) and (30), getting $f_{E K} \approx-1.50549949 T$; thus, the residual entropy becomes $\mathcal{S} \approx 1.50549949$, as expected independent of temperature. This result complies with the frustrated region found in reference [29]. We recognized that Heisenberg spin exchange interaction influences the frustrated (FRU) phase, but the origin of frustration and the magnitude arise in a similar way to antiferromagnetic Ising Kagomé lattice 29].

Second, the condition for $\frac{w_{3}}{w_{1}}=r=1$ and $T \rightarrow 0$, occurs in the interface of QFI and FRU states, so we have $A(\phi)=2^{5}$ and $Q(\phi)=2^{10}$; thus the free energy reduces to

$$
f_{E K}=-2 T \ln \left(w_{1}\right)-\frac{T}{4 \pi} \int_{0}^{2 \pi} \ln \left(2^{6}\right) \mathrm{d} \phi,
$$

this implies that the residual entropy merely becomes as $\mathcal{S}=3 \ln (2) \approx 2.07944$.

Third, when $w_{3}>w_{1}$ and $T \rightarrow 0$, then $r \rightarrow \infty$, so we have

$$
\begin{aligned}
& A(\phi) \approx 2 r^{3} \cos (\phi)+\frac{1}{2} r^{4} \sim \frac{1}{2} r^{4}, \\
& Q(\phi) \approx \frac{1}{4} r^{8}-8 r^{6}(1+\cos (\phi)) \sim \frac{1}{2} r^{8} .
\end{aligned}
$$

Consequently, the free energy reduces to

$$
\begin{aligned}
f_{E K} & \approx-2 T \ln \left(w_{1}\right)-\frac{T}{4 \pi} \int_{0}^{2 \pi} \ln \left(\frac{1}{2} r^{4}+\sqrt{\frac{1}{4} r^{8}}\right) \mathrm{d} \phi, \\
& \approx-2 T \ln \left(w_{1}\right)-2 T \ln \left(\frac{w_{3}}{w_{1}}\right), \\
& \approx-2 T \ln \left(w_{3}\right) .
\end{aligned}
$$

In addition, as expected, there is no frustration in this region $\mathrm{FM}(\mathrm{FIM})$ or QFI because there is no residual entropy $(\mathcal{S}=0)$ when $T=0$.

Once the free energy is obtained, we can analyze the thermodynamic properties of the expanded Kagomé Ising-Heisenberg model.

\section{A. Critical temperature}

In the following, we will discuss one of the essential properties of the two-dimensional lattice models, the critical behavior of temperature. It is well established that the critical temperature for the Kagomé lattice is given by $\frac{K}{T_{c}}=\ln (3+2 \sqrt{3})[12,32]$. As an alternative, we

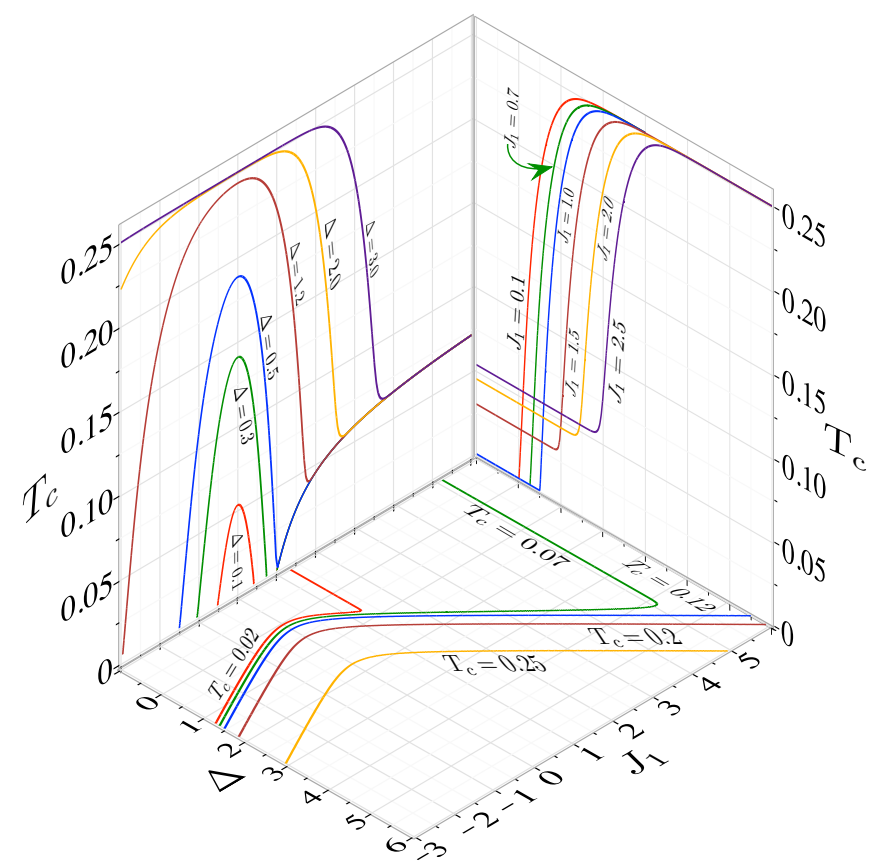

Figure 5: (Left panel) Critical temperature $T_{c}$ against $J_{1}$, for several values of $\Delta$. (Right panel) Critical temperature $T_{c}$ as a function of $\Delta$, for a range of values in $J_{1}$. (Bottom) The phase diagram in the plane $\Delta-J_{1}$ for several critical temperature values. In all panels a fixed $J_{2}=1$ was considered.

are able to write the critical temperature as a ratio of Boltzmann factor:

$$
\frac{w_{3}^{c}}{w_{1}^{c}}=r_{c}=3+2 \sqrt{3}
$$

where $w_{1}^{c}$ and $w_{3}^{c}$ refer to the Boltzmann factors at a critical temperature, which is given by (19) and (20) at $T=T_{c}$, respectively.

After some algebraic manipulation, this condition can be rewritten more explicitly as

$$
z_{c}^{4}=\frac{\left(x_{c}^{6}+2\right)(3+2 \sqrt{3})-1-\frac{y_{c}^{-1} v_{+}^{c}+y_{c} v_{-}^{c}}{x_{c}^{-3}\left(y_{c}+y_{c}^{-1}\right)}}{x_{c}^{2}\left(1-\left(y_{c}^{2}-1+y_{c}^{-2}\right)(3+2 \sqrt{3})\right)} .
$$

In Fig $\left[\right.$ (left) is illustrated the critical temperature $T_{c}$ as a function of $J_{1}$ for several values of $\Delta$ and fixing $J_{2}=1$. The dashed line corresponds to $\Delta=0.5$, which is a special curve with two critical points at zero temperature (for detail see Fig 2). For larger value of $\Delta>0.5$ only occurs one critical point at zero temperature, while for $0<\Delta<0.5$, there are three critical points at zero temperature. However, for $\Delta<0$ only appears one critical point at zero temperature, which always occurs at $J_{1}=1$ and is independent of $\Delta$. This curve agrees with the zero temperature phase diagram shown in Fig. 2 
Similarly, Fig 5 (right) shows the critical temperature $T_{c}$ as a function of $\Delta$ and for several values of $J_{1}$. For $\Delta<0$, the critical temperature is independent of $\Delta$. Likewise, for $\Delta \rightarrow \infty$, the critical temperature also becomes independent of $\Delta$, and in this limit, we have $T_{c} \rightarrow 0.25$. While, in Fig 5 (bottom) the phase diagram at fixed critical temperature $T_{c}$ in the $J_{1}-\Delta$ plane is reported and for different critical temperature $T_{c}$ values. When $T_{c} \rightarrow 0$, this phase diagram leads to a zero temperature phase diagram (see the solid line in Fig [2), since there is no evidence of phase transition between FM and QFI phase at zero temperature. It is worth noting that, for $J_{2}=-1$, the curves become identical, the only difference is that, instead of FM phase we have an FIM phase.

Using this expression, we can obtain the critical temperature in the limit of $J_{1} /\left|J_{2}\right| \rightarrow \infty$, leading to

$$
T_{c}=\frac{\left|J_{2}\right|}{6 \ln (2+\sqrt{3}+\sqrt{6+4 \sqrt{3}})}=0.0836826082\left|J_{2}\right| .
$$

While for $\Delta /\left|J_{2}\right| \rightarrow \infty$, the critical temperature leads to $T_{c} /\left|J_{2}\right|=0.25$.

\section{B. Spontaneous Magnetization}

Another relevant quantity to analyze here is the spontaneous magnetization of the present model. Therefore, it is pertinent to examine the magnetization of the expanded Kagomé Ising-Heisenberg lattice. The Ising spin magnetization $m_{I}=\langle s\rangle$, can be determined concerning the effective Kagomé Ising lattice magnetization, while the magnetization of Heisenberg spins can be obtained using the decoration transformation approach $[11,13,15]$

$$
\left\langle\sigma_{1}^{z}\right\rangle=\eta\left\langle s_{1}\right\rangle+\frac{\gamma}{3}\left\langle s_{1} s_{2} s_{3}\right\rangle
$$

which linearly combines single and triple Ising spin average $\langle s\rangle$ and $\left\langle s_{1} s_{2} s_{3}\right\rangle$.

To find the coefficients $\eta$ and $\gamma$ we use the following relation [11 13, 15]

$$
\zeta(\{s\})=\left[\eta\left(s_{1}+s_{2}+s_{3}\right)+\gamma s_{1} s_{2} s_{3}\right] w(\{s\}) .
$$

In a similar way, we define

$$
\tilde{\zeta}(\{s\})=\operatorname{tr}_{\{\sigma\}}\left[\left(\sum_{i=1}^{3} \sigma_{i}^{z}\right) \mathbf{V}(\{\boldsymbol{\sigma}, s\})\right] .
$$

On the other hand, the spin coupling configurations $\{\uparrow \uparrow \uparrow\}$ and $\{\uparrow \uparrow \downarrow\}$, is denoted merely as $\tilde{\zeta}(1 / 2)=\tilde{\zeta}_{1}$ and $\zeta(3 / 2)=\zeta_{3}$. Therefore, we have

$$
\begin{aligned}
& \tilde{\zeta}_{3}=\operatorname{tr}_{\{\sigma\}}\left[\mathbf{U}_{3}\left(\sum_{i=1}^{3} \sigma_{i}^{z}\right) \mathbf{U}_{3}^{-1} \mathbf{D}_{3}\right], \\
& \tilde{\zeta}_{1}=\operatorname{tr}_{\{\sigma\}}\left[\mathbf{U}_{1}\left(\sum_{i=1}^{3} \sigma_{i}^{z}\right) \mathbf{U}_{1}^{-1} \mathbf{D}_{1}\right],
\end{aligned}
$$

where $\mathbf{D}_{3}$ and $\mathbf{D}_{1}$ are the diagonalized matrix representation of $\mathbf{V}$ for each sector, and these are given by

$$
\begin{gathered}
\mathbf{D}_{3}=\operatorname{diag}\left\{\frac{3}{2} y^{3} z^{3}, \frac{-1}{2 z y x^{2}}, \frac{-1}{2 z y x^{2}}, \frac{x^{4}}{2 z y}, \frac{-x^{4}}{2 z y},\right. \\
\left.\frac{y}{2 z x^{2}},-\frac{3}{2} y^{-3} z^{3}\right\}
\end{gathered}
$$

and

$$
\begin{gathered}
\mathbf{D}_{1}=\operatorname{diag}\left\{\frac{3}{2} y z^{3}, \frac{1}{2 z y x^{2}}, \frac{x y}{2 z v_{-}}, \frac{x y v_{-}}{2 z},-\frac{x}{2 z y v_{+}}, \frac{-x v_{+}}{2 z y},\right. \\
\left.\frac{y}{2 z x^{2}},-\frac{3}{2} y^{-1} z^{3}\right\} .
\end{gathered}
$$

The orthogonal matrices $\mathbf{U}_{3}$ and $\mathbf{U}_{1}$ are obtained straightforwardly for each configuration $\{\uparrow \uparrow \uparrow\}$ and $\{\uparrow \uparrow \downarrow\}$ respectively, which are expressed below

$$
\mathbf{U}_{3}=\left(\begin{array}{cccccccc}
1 & 0 & 0 & 0 & 0 & 0 & 0 & 0 \\
0 & 0 & 0 & 1 & 0 & -1 & -\frac{1}{2} & 0 \\
0 & 0 & 0 & 1 & 0 & 1 & -\frac{1}{2} & 0 \\
0 & -1 & -\frac{1}{2} & 0 & 1 & 0 & 0 & 0 \\
0 & 0 & 0 & 1 & 0 & 0 & 1 & 0 \\
0 & 0 & 1 & 0 & 1 & 0 & 0 & 0 \\
0 & 1 & -\frac{1}{2} & 0 & 1 & 0 & 0 & 0 \\
0 & 0 & 0 & 0 & 0 & 0 & 0 & 1
\end{array}\right)
$$

and

$$
\mathbf{U}_{1}=\left(\begin{array}{cccccccc}
1 & 0 & 0 & 0 & 0 & 0 & 0 & 0 \\
0 & 0 & u_{1,-} & u_{1,+} & 0 & 0 & 0 & 0 \\
0 & -1 & 1 & 1 & 0 & 0 & 0 & 0 \\
0 & 0 & 0 & 0 & -1 & 1 & 1 & 0 \\
0 & 1 & 1 & 1 & 0 & 0 & 0 & 0 \\
0 & 0 & 0 & 0 & 1 & 1 & 1 & 0 \\
0 & 0 & 0 & 0 & 0 & u_{2,-} & u_{2,+} & 0 \\
0 & 0 & 0 & 0 & 0 & 0 & 0 & 1
\end{array}\right)
$$

where $u_{1, \pm}=-\frac{1}{2}+\frac{J_{2}}{J_{1}} \pm \frac{J_{-}}{2 J_{1}}$ and $u_{2, \pm}=-\frac{1}{2}-\frac{J_{2}}{J_{1}} \pm \frac{J_{+}}{2 J_{1}}$.

Consequently, the relations (41) and (42) after some algebraic manipulation, simply becomes as

$$
\begin{aligned}
\tilde{\zeta}_{3}= & \frac{3}{2} z^{3}\left(y^{3}-y^{-3}\right)+z^{-1}\left(\frac{1}{2} x^{4}+x^{-2}\right)\left(y-y^{-1}\right), \\
\tilde{\zeta}_{1}= & \left(y-y^{-1}\right)\left(\frac{3}{2} z^{3}-\frac{1}{2} x^{-2} z^{-1}\right) \\
& +\frac{1}{2}\left(v_{-} y-v_{+} y^{-1}\right) x z^{-1} .
\end{aligned}
$$

From Eq. (39) we also have the following relations

$$
\begin{aligned}
\zeta_{3} & =\left(\frac{3}{2} \eta+\frac{1}{8} \gamma\right) w_{3}, \\
\zeta_{1} & =\left(\frac{1}{2} \eta-\frac{1}{8} \gamma\right) w_{1} .
\end{aligned}
$$

From where we obtain the unknown coefficients

$$
\begin{aligned}
& \eta=\frac{1}{2}\left(\frac{\zeta_{3}}{w_{3}}+\frac{\zeta_{1}}{w_{1}}\right), \\
& \gamma=2\left(\frac{\zeta_{3}}{w_{3}}-3 \frac{\zeta_{1}}{w_{1}}\right),
\end{aligned}
$$


where $\zeta_{3}=\tilde{\zeta}_{3}$ and $\zeta_{1}=\tilde{\zeta}_{1}$, with $\tilde{\zeta}_{3}$ and $\tilde{\zeta}_{1}$ given by Eqs. (47) and (48).

Using the relations for the correlation functions obtained by Barry et al.[32] (see Eq.(2.9a) of Ref. [32]). Now we need to relate the three spin thermal average and single spin average

$$
\left\langle s_{1} s_{2} s_{3}\right\rangle=R(r)\left\langle s_{1}\right\rangle
$$

where $R(r)$ after some algebraic manipulation becomes

$$
R(r)=\frac{r^{3}-3 r^{2}-r-5}{4(r-1)^{3}} .
$$

Moreover, the single Heisenberg spins average can be written as

$$
\left\langle\sigma^{z}\right\rangle=\left[\eta+\frac{\gamma}{3} R(r)\right]\left\langle s_{1}\right\rangle .
$$

On the other hand, the thermal average of Ising spin $\left\langle s_{1}\right\rangle[32]$ is given by

$$
\left\langle s_{1}\right\rangle=\left(r^{2}-6 r-3\right)^{\frac{1}{8}} G(r),
$$

where $G(r)$ is defined as in reference [32], For the present model $G(r)$ has been adapted as a function of $r$, which is expressed as

$$
G(r)=\left(\frac{r+3}{r-1}\right)^{\frac{1}{4}} \frac{\left(r^{2}+2 r+5\right)^{\frac{3}{8}}}{2(r+1)} .
$$

We emphasize that $G(r)$ is always a positive amount. Furthermore, the factor 2 in the denominator is included because we are considering the Ising spin eigenvalues as $\pm 1 / 2$. For our case, the thermal average of Ising spin and Heisenberg spin, are defined as $m_{I}=\langle s\rangle$ and $m_{H}=\langle\sigma\rangle$ respectively.

Notice that the magnetization exponent satisfies the same universality class of that Kagomé lattice (same critical exponent $1 / 8)$.

Hence, the total magnetization per spin can be expressed using the Eq.([6]), so we have

$$
m_{t}=\frac{\langle s\rangle+2\left\langle\sigma^{z}\right\rangle}{3} .
$$

In Fig 6 is illustrated the magnetization of Ising spin as a function of $J_{1}$ and $\Delta$, considering fixed parameter $J_{2}=1$ and temperature $T=0.01$. Fig $[6$ a displays the Ising spin magnetization, and we observe the regions FM and QFI have the magnetization leading to $\langle s\rangle \rightarrow 0.5$, whereas in FRU region the magnetization becomes null. In the interface between FM and QFI, there is no spontaneous magnetization for Ising spin, this means there is no phase transition at finite temperature, but there is only a zero temperature phase transition.

Analogously Fig $6 \mathrm{~b}$ displays Heisenberg spin magnetization, here we can see clearly the magnetization in region FM leads to $\langle s\rangle \rightarrow 0.5$, while the magnetization in (a)

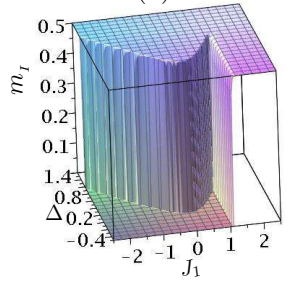

(b)

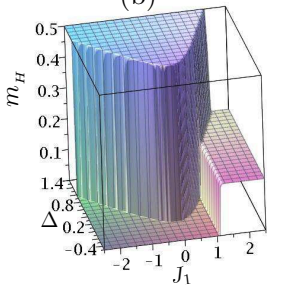

(c)

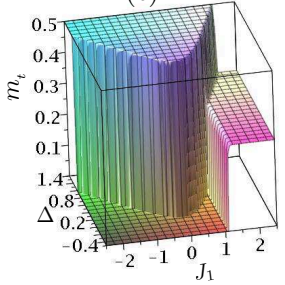

Figure 6: Magnetization as a function of $J_{1}$ and $\Delta$, assuming $T=0.01$ and $J_{2}=1$. (a) Ising spin magnetization. (b) Heisenberg spin magnetization. (c) Total spin magnetization per unit lattice.
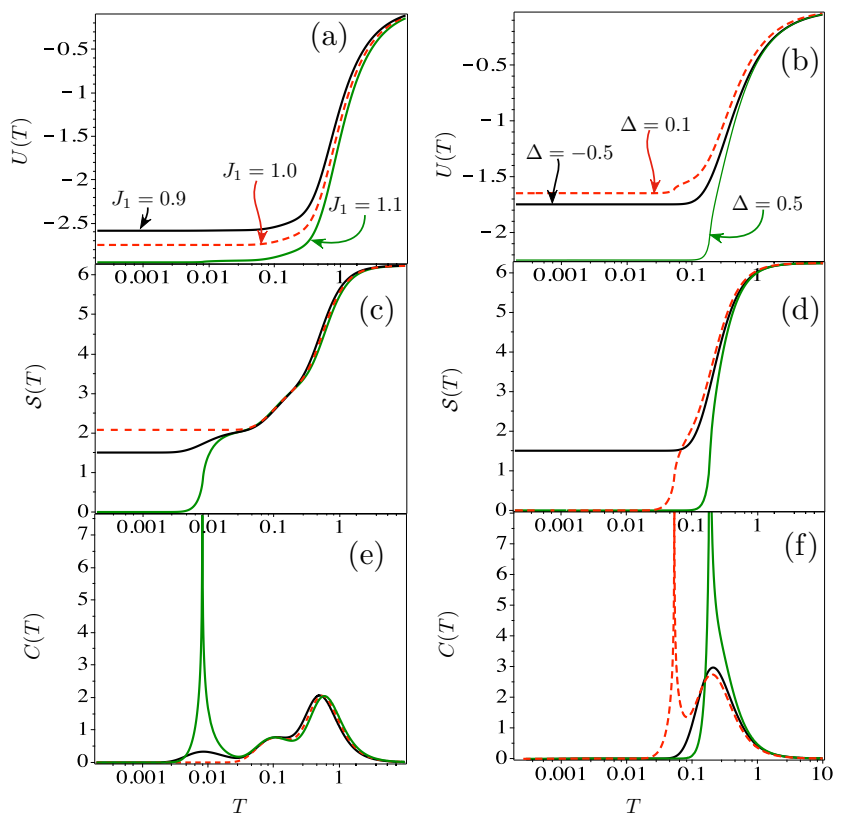

Figure 7: In left panel is considered fixed parameters $\Delta=$ -0.5 and $J_{1}$ is given in (a). In right panel is assumed fixed $J_{1}=0.0$ and $\Delta$ is given in (b). (a-b) Internal energy as a function of temperature for fixed parameters. (c-d) Entropy as a function of temperature. (e-f) Specific heat as a dependence of temperature.

QFI region becomes $\langle s\rangle \rightarrow 1 / 6$, and naturally, in FRU region the magnetization becomes null. Whereas the total magnetization is depicted in Fig 6r: thus in region FM $m_{t} \rightarrow 0.5$, in QFI region $m_{t} \rightarrow 1 / 3$ and for FRU region the total magnetization readily is null.

\section{Entropy and specific heat}

To complete our investigation concerning this model, let us discuss the thermodynamic properties, which can be obtained straightforwardly from the free energy $f_{E K}$, such as entropy $\mathcal{S}=-\partial f_{E K} / \partial T$, internal energy $U=$ $f_{E K}+T \mathcal{S}$ and specific heat $C=T \partial \mathcal{S} / \partial T$.

In Fig [7a is displayed the internal energy as a function of temperature considering fixed parameter $J_{1}$ as 
described in the panel (a): for $J_{1}=0.9$ and $J_{1}=1.0$ the curves increases smoothly, but $J_{1}=1.1$ there is a tiny jump at $T_{c} \approx 0.082$. In Fig $7 \mathrm{~b}$ is depicted the internal energy as a dependence of temperature, for $\Delta=0.5$ and $\Delta=0.1$ there is a strong change of curvature at $T_{c} \approx 0.186$ and $T_{c} \approx 0.0537$ respectively, whereas for $\Delta=-0.5$ there is no evidence of phase transition at finite temperature. The next panel (c) in Fig.7 reports the entropy for the same set of parameters as in (a), for $J_{1}=0.9$ the residual entropy leads to $\mathcal{S}=1.5055$, while for $J_{1}=1.0$ (equivalently $r=1$ ) also exhibits another residual entropy given by $\mathcal{S}=3 \ln (2)=2.07944$, but for $J_{1}=1.1$ there is no residual entropy because the system leads to QFI phase. Similarly, in Fig 77d we show the entropy as a function of temperature for the set of parameters of the panel (b). Once again for $\Delta=0.5$ and $\Delta=0.1$, there is a sudden change close to the critical temperature, but for $\Delta=-0.5$ the entropy increases smoothly indicating the absence of phase transition. Finally, in Fig 7f confirms the critical temperature for the same set of parameters described in panel (b) as a divergence in specific heat.

\section{CONCLUSIONS}

Several materials have exotic structures such as honeycomb lattice, a triangular lattice, Kagomé lattice among others. Hence it is worth to investigate the magnetic properties and geometric frustration of these kinds of models. Therefore, in this work was considered the IsingHeisenberg model on expanded Kagomé lattice, that we could also name as triangle-dodecagon (3-12) lattice, this model can even be viewed as a decorated star lattice. Considering all spins located in triangles as Heisenberg spins while remaining spins are of Ising type spins. Consequently, this model is equivalent to an effective Kagomé Ising lattice, through a direct decoration transformation technique[15]. This model allows us to study zero temperature magnetic properties of the 3-12 lattice, such as the phase diagram at zero temperature, where we found four phases, a frustrated (FRU) phase, a ferromagnetic (FM) phase, a classical ferrimagnetic (FIM) phase and a quantum ferrimagnetic (QFI) phase. We remarked that Heisenberg spin exchange interaction strongly influences the frustrated phase; however, we rigorously verified that the magnitude and origin of the frustration turn out in the same fashion as that of the antiferromagnetic Ising Kagomé lattice.[29]. We also obtain the free energy of the model which permit us to explore, the critical temperature and the spontaneous magnetization were also considered as a dependence of Hamiltonian parameters. In addition, we have investigated the entropy where we observed a residual entropy in the frustrated region $\mathcal{S} \approx 1.5055$. As well as in the interface between QFI and FRU the residual entropy is given by $\mathcal{S}=3 \ln (2)$. Besides, we also studied the specific heat divergence as a function of temperature to handle the phase transition.

\section{Acknowledgments}

This work was partially supported by Brazilian agencies FAPEMIG, CAPES and CNPq.
[1] L. Onsager, Phys. Rev. 65, 117 (1944).

[2] T. Horiguchi, Phys. Lett. A 113, 425 (1986).

[3] M. Kolesik and L. Samaj, Int. J. Mod. Phys. B 6, 1529 (1992).

[4] F. Y. Wu, J. Math. Phys. 15, 687 (1974); J. Phys. A: Math Gen. 23, 375 (1990).

[5] P. Azaria and H. Giacomini, J. Phys. A: Math. Gen. 21, L935 (1988).

[6] W. T. Lu and F. Y. Wu, Phys. Rev. E 71, 046120 (2005).

[7] Shores, M. P.; Nytko, E. A.; Bartlett, B. M.; Nocera, D. G. J. Am. Chem. Soc. 127, 13462 (2005)

[8] Han, T. H.; Helton, J. S.; Chu, S. Y.; Nocera, D. G.; Rodriguez- Rivera, J. A.; Broholm, C.; Lee, Y. S. Nature 492, 406 (2012)

[9] J. A. Plascak, V.K. Varma, Journ. Magn. and Mag. Mat. 468, 224 (2018)

[10] M. Ertaş, E. Kantar, M. Keskin, Journ. Magn. and Mag. Mat. 358, 56 (2014)

[11] M. E. Fisher, Phys. Rev. 113, 969 (1959).

[12] I. Syozi, in Phase Transitions and Critical Phenomena, edited by C. Domb and M. S. Green (Academic Press, New York, 1972), Vol. 1.

[13] O. Rojas, J. S. Valverde and S. M. de Souza, Physica A 388, 1419 (2009).
[14] J. Strecka, Phys. Lett. A, 374, 3718 (2010) ; LAP LAMBERT Academic Publishing, Saarbrucken, Germany, 2010, ISBN: 978-3-8383-6200-7.

[15] O. Rojas and S. M. de Souza, J. Phys. A: Math Theor. 44, 245001 (2011).

[16] M. Rojas, O. Rojas, S. M. de Souza, Phys. Rev. E 86, 051116 (2012).

[17] M. Blume, V. J. Emery and R. B. Griffiths, Phy. Rev. A 4, 1071 (1971).

[18] F.Y. Wu, Phys. Lett. A 116, 245 (1986).

[19] J. W. Tucker, J. Appl. Phys. 69, 6164 (1991).

[20] V. Urumov, J. Phys. C: Solid State Phys. 20, L875 (1987).

[21] Y. L. Loh, D. X. Yao and E. W. Carlson, Phys. Rev. B 77, 134402 (2008).

[22] J. Strecka, L. Canova, M. Jascur and M. Hagiwara, Phys. Rev. B 78, 024427 (2008); J. Cisarova, J. Strecka Phys. Rev. B, 87 (2013), 024421; J. Cisarova, F. Michaud, F. Mila, J. Strecka, J. Phys. Rev. B. 87 (2013) 054419.

[23] K. Y. Lin and T. L. Chen, Chin. J. Phys. 25, 178 (1987).

[24] K. Y. Lin and J. L. Chen, J. Phys. A: Math. Gen. 20, 5695 (1987).

[25] J. H. Barry and M. Khatun Phys. Rev. B 51, 5840 (1995).

[26] J. S. Valverde, O. Rojas, and S. M. de Souza, Phys. Rev. 
E 79, 041101 (2009).

[27] Y. Tang, C. Peng, W. Guo, J. Wang, G. Su, and Z. He, J. Am. Chem. Soc. 139, 14057 (2017).

[28] Y. Z. Zheng, M. L. Tong, W. Xue, W. X. Zhang, X.-M. Chen, F. Grandjean, and G. J. Long, Angew Chem Int Ed Engl 46, 6076 (2007).

[29] K. Kano and S. Naya, Prog. Theor. Phys. 10, 158 (1953).
[30] P. Azaria, H. T. Diep and H. Giacomini, Phys. Rev. Lett., 59,1629 (1987).

[31] C. Fan and F. Wu, Phys. Rev. B 2, 723 (1970).

[32] J. H. Barry, T. Tanaka, M. Khatun and C. H. Munera, Phys. Rev. B 442595 (1991). 